\title{
MODELAGEM DE MUDANÇA DO USO DA TERRA NO NOROESTE DO MATO GROSSO: POSSIBILIDADES DE ANÁLISE AMBIENTAL A PARTIR DA REVOLUÇÃO DA GEOGRAFIA QUANTITATIVA.
}

Max Paulo Rocha Pereira ${ }^{1}$

\begin{abstract}
Resumo:
A evolução do pensamento geográfico possibilitou o desenvolvimento da geografia quantitativa que conferiu aos estudos dentro do escopo das ciências geográficas um maior rigor metodológico, aproximando a geografia do que vários autores chamaram de neopositivismo. Com a evolução dos softwares, criação de metodologias e aumento da capacidade de processamento foi possível dar mais robustez as análises e garantiu ainda à possibilidade de elaboração de modelos analíticos e preditivos representando fenômenos ambientais e antrópicos em diferentes escalas com grau de similaridade cada vez maior. Como estudo de caso, este trabalho buscou apresentar dados do projeto PRODES do INPE, além de realizar uma modelagem preditiva do desmatamento na região noroeste do Mato Grosso. Os dados revelaram um acréscimo no desmatamento na região para 2033 caracterizado pela mudança de uso da terra na área analisada.
\end{abstract}

\section{ABSTRACT:}

The geographic thinking evolution enabled the quantitative geography development, conferring the studies inside the geographical sciences scope superior methodological severity, approaching the geography to the denominated by several authors neopositivism. Along with software evolution, the development of methodologies and the increase of the processing capacity it was possible to increase the robustness in the analyzes and guarantee the possibility of analytical and predictive models elaboration representing environmental and anthropic phenomena in different scales and better degree of similarity. As a case study, this work sought to present data from INPE's PRODES project, in addition to perform the predictive modeling for deforestation in the northwest region of Mato Grosso. The data revealed an increase in deforestation in the region for 2033 characterized by the land use change in the analyzed area.

1 Universidade Federal de Minas Gerais, Departamento de Geografia, Belo Horizonte, MG, Brasil mqualidadeambiental@gmail.com 


\section{1 - INTRODUÇÃO}

A ciência contemporânea busca, através do estudo de seus métodos, a elaboração de modelos conceituais e específicos, que possibilitem aos seus diferentes campos do saber, a identificação e caracterização de um, ou mais, objetos de estudo. Armond e Afonso (2011) ao estudar a geografia física a partir de uma ótica integradora, destacam que, nesse período em que a ciência questiona seu estatuto epistemológico moderno, uma das questões que se coloca enquanto fruto desse movimento é um resgate da história da ciência.

Neste sentido é importante ao elaborarmos um estudo com o recorte geográfico compreender a historicidade desta ciência e as transformações que a mesma passou até o presente. Se tratando da Geografia e sua episteme a partir da década de 1950, muito do que havia sido postulado pela "Geografia clássica" estava sendo repensado a partir de uma percepção da inutilidade das observações e dos resultados obtidos a partir da suas técnicas.

Esse movimento do repensar o arcabouço metodológico, as questões a serem respondidas e até mesmo a forma das respostas, deu origem no que chamamos de revolução quantitativa. As idéias e abordagens difundidas durante a década de 50, eram ainda influenciadas por um contexto de pós segunda guerra mundial, onde o surgimento de novas demandas, novas configurações espaciais e principalmente o estabelecimento de novas relações internacionais desencadearam uma profusão de idéias que no campo da geografia orientou o surgimento de sua revolução quantitativa e teorética.

Além da adoção de maior rigor metodológico, Christofoletti (1985, p.18) aponta para apropriação do conceito sistêmico no campo da geografia e discorre sobre a importância da obtenção do conhecimento teórico conceitual acerca do problema em análise, uma vez que estudos realizados por pesquisadores que não possuam o embasamento necessário para sua interpretação corre um sério risco, por mais evoluído que seja o método analítico de ser apenas um trabalho mecanizado, não representando um trabalho geográfico.

Kwan e Schwanen (2009) destacam um segundo momento da geografia quantitativa que foi marcado pela utilização das geotecnologias e evolução dos Sistemas de Informação Geográficas (SIGs), que possibilitaram maior velocidade, trabalhar em varias escalas em contraponto a regionalização utilizada pela geografia clássica e principalmente a formulação de modelos que permitissem aos geógrafos uma análise preditiva acerca da relação de características naturais/físicas aliadas a influência antrópica.

Sobre essa possibilidade Christofoletti (1982, p. 19-20) considera que a construção de modelos é uma abstração da realidade a partir de uma estruturação sequencial dos mecanismos de 
funcionamento de um dado sistema, tanto para torná-lo mais compreensível como para demonstrar as relações existentes entre seus diferentes componentes.

A compreensão das alterações no uso da terra em escala temporal é de fundamental importância para o estabelecimento de uma boa gestão do espaço. Isto porque as alterações causadas pela ação antrópica possuem potencial para modificar significativamente a paisagem. Logo, desenvolver métodos e metodologias que promovam uma análise dessas paisagens, assumindo as ações antrópicas como variáveis em modelos preditivos podem, acima de tudo, subsidiar a tomada de decisão por parte de gestores públicos e sociedade como um todo. E, consequentemente promover uma melhor utilização dos recursos naturais e do espaço/território.

Partindo desse referencial foi desenvolvida diferentes técnicas e formas de utilizar a modelagem nos estudos geográficos nas mais diversas escalas. E essas possibilidades só se concretizaram a partir do aprofundamento das ciências geográficas no conhecimento de métodos estatísticos tanto na obtenção como no tratamento de dados e na evolução dos softwares e da capacidade de processamento dos equipamentos.

No Brasil várias foram as utilizações das técnicas de análise a partir da evolução do pensamento geográfico, do aprimoramento da geografia quantitativa e principalmente da elaboração de modelos representativo dos sistemas ambientais. Um projeto que merece destaque e que é objeto de estudo deste trabalho foi o fruto do trabalho do Instituto de Pesquisas Espacial (INPE) com financiamento do Ministério da Ciência Tecnologia, Inovação e Comunicações (MCTIC).

O projeto nomeado PRODES realiza o monitoramento por satélite do desmatamento por corte raso na Amazônia Legal, produzindo desde 1988, um relatório acerca das taxas anuais de desmatamento na região. Várias são as possibilidades de utilização desses dados, dentre os quais se destacam a formulação de políticas públicas para fiscalização e controle e a elaboração de modelos preditivos do desmatamento na região.

Analisar a mudança no uso e cobertura da terra a partir de série histórica de dados e simulação futura apresenta então o potencial de subsidiar o ordenamento do uso de determinada região. Uma das técnicas mais utilizadas neste tipo de análise consiste na atribuição de pesos as variáveis que influenciam no processo de mudança no uso da terra que associadas a uma matriz de transição histórica da mudança observada, possibilita uma simulação de cenário ao longo dos anos.

O Brasil possui um dos maiores extratos de floresta tropical já conhecido, assim como significativa biodiversidade. No entanto, em decorrência da alta taxa de desmatamento, da fragmentação de hábitats promovida pela urbanização e industrialização e o aumento constante da fronteira agrícola 
dentre outros fatores tem levantado discussões e estudos acerca da perda dessa biodiversidade e suas consequências no equilíbrio dos ecossistemas.

Este trabalho objetivou analisar dados do projeto PRODES além de realizar a modelagem utilizando a técnica de autômato celular estocástico de mudança no uso da terra para a região noroeste do Mato Grosso. Com o propósito de avaliar o aumento do desmatamento na referida região em uma janela de 6 anos observados e 30 anos simulados, discutindo assim as possibilidades de análise ambiental a partir das técnicas advindas da evolução da geografia quantitativa.

\section{2 - MATERIAIS E MÉTODO}

Para compreender melhor sobre a evolução do pensamento geográfico mais especificamente acerca da revolução da geografia quantitativa, fez-se necessário a realização de extensa pesquisa bibliográfica em periódicos e livros que sustentaram a fundamentação e a discussão deste artigo. O estudo utilizando os dados do PRODES foi realizado a partir de pesquisa documental entre os anos de 2004 a 2017 e posteriormente submetido a método de processamento específico para os anos anteriores (1997 a 2003) e projeção do desmatamento para o ano de 2033.

O recorte em análise corresponde à cena do projeto PRODES de órbita 229, ponto 67, correspondente à região noroeste do estado do mato grosso, inserida na mesorregião norte matogrossense, compreendendo parte dos municípios de Colniza, Cotriguaçu, Nova Bandeirantes, Juara, Castanheira, Juína, Aripuanã e Juruena como pode ser observado na Figura 1. 
Figura 1: Localização da Área de Estudo

\section{Localização da Área de Estudo}

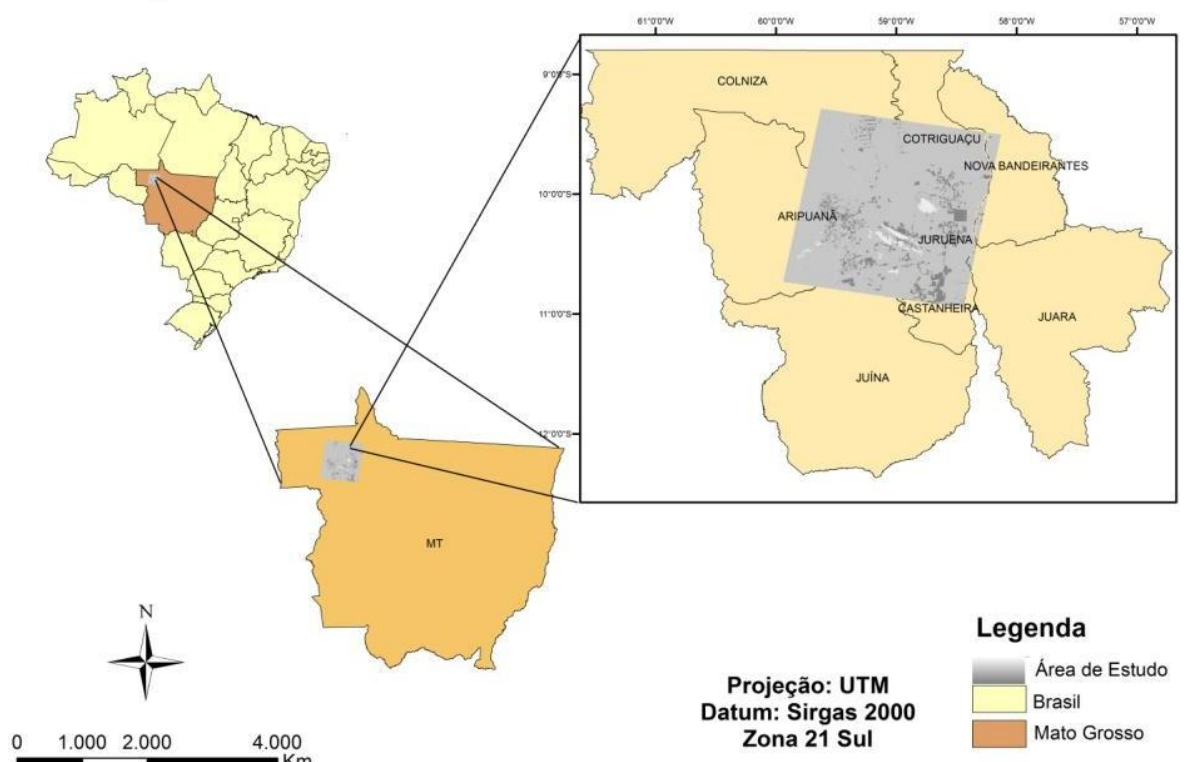

Fonte: Autores

A área possui uma economia predominantemente agrícola, em processo ativo e crescente de urbanização, que, no entanto mantém ainda considerável reserva florestal. Sendo assim, representa uma região de relevante interesse socioambiental por se inserir em um contexto de pressão intensa de expansão da fronteira agrícola nacional, sofrendo influência tanto da industrialização e consequentemente a urbanização, quanto do desenvolvimento agrícola (FRANÇA, 2009).

As oito cidades sobre as quais esta inserida a cena em análise possuem juntas um total de 184.535 habitantes, com predominância de Argissolos, com estrato vegetacional predominante no bioma amazônico apresenta baixo adensamento urbano, no entanto intensiva atividade agrícola.

Para realização da modelagem e processamento dos dados foram utilizados os softwares Dinamica EGO versão 3.0.17 e o Arcgis versão 10.3 (ESRI, 2013). A metodologia para elaboração do modelo espaço/temporal adotou os seguintes passos, a saber: (a) pré- processamento, (b) cálculo da matriz de transição com série histórica de dados, (c) cálculo dos pesos de evidência (variáveis categóricas), (d) cálculo das variáveis contínuas, (e) correlação dos mapas, (f) simulação, (g) cálculo de nova matriz de transição para validar o modelo (capacidade de predição) e (h) projeção das trajetórias. 
No pré-processamento foi executada a padronização e rasterização das variáveis utilizadas. $\mathrm{Na}$ sequencia procedeu-se o cálculo da matriz de transição no software Dinamica EGO, através da tabulação cruzada dos mapas de uso inicial (1997) e final (2003).

Em seguida foram executados os cálculos dos intervalos e dos pesos de evidência, respectivamente para as seguintes variáveis: a) às distâncias; b) às variáveis estáticas e; c) às classes de uso do solo. Os pesos foram então estimados com base na independência dos eventos (Teorema de Bayes) e analisados de acordo com os resultados obtidos do teste de Crammer, excluindo-se variáveis com índice acima de 0.5 .

Para a parametrização do modelo, foram utilizados os algoritmos patcher e expander que são responsáveis pela alocação de mudanças de cobertura e uso da terra. A função expander calcula a expansão de manchas previamente existentes de uma determinada classe. A função patcher, por sua vez, calcula o surgimento de novas manchas (SOARES-FILHO et al., 2002). Em seguida, executaram-se as simulações, adotando-se índice de isometria igual a 2.0.

$\mathrm{Na}$ fase de validação, utilizou-se o índice de similaridade fur:̧y, e para tal adotou-se o decaimento constante com os seguintes tamanhos de janelas: $1 \times 1,3 \times 3,5 \times 5,7 \times 7,9 x 9$ e 11x11, na sequencia avaliou-se os cenários de desmatamentos para o ano de 2033.

\section{3 - DISCUSSÃO DOS RESULTADOS}

O monitoramento do PRODES permite a identificação de períodos críticos do desmatamento da Amazônia nos diferentes estados que apresentam a fitofisionomia do bioma Amazônico, sendo, portanto, essencial instrumento de fiscalização e controle. Como pode ser observado na tabela 1, o estado do Mato Grosso embora tenha diminuído a taxa de desmatamento nos últimos anos, ainda apresenta a segunda maior perda de área de Floresta Amazônica desmatada em comparação com os outros estados analisados, perdendo apenas pro Pará, com quem faz fronteira e divide problemas relacionados à grilagem, estrutura fundiária deficiente e dificuldades de controle legal do desmatamento. 
Tabela 1: Taxa PRODES 2004 a $2017\left(\mathrm{~km}^{2}\right)$

\begin{tabular}{ccccccccccc}
\hline Ano & AC & AM & AP & MA & MT & $\begin{array}{c}\text { PA } \\
\mathbf{k m}^{2}\end{array}$ & RO & RR & TO & Amz Legal \\
\cline { 2 - 10 } 2004 & 728 & 1232 & 46 & 755 & 11814 & 8870 & 3858 & 311 & 158 & 27772 \\
2005 & 592 & 775 & 33 & 922 & 7145 & 5899 & 3244 & 133 & 271 & 19014 \\
2006 & 398 & 788 & 30 & 674 & 4333 & 5659 & 2049 & 231 & 124 & 14286 \\
2007 & 184 & 610 & 39 & 631 & 2678 & 5526 & 1611 & 309 & 63 & 11651 \\
2008 & 254 & 604 & 100 & 1271 & 3258 & 5607 & 1136 & 574 & 107 & 12911 \\
2009 & 167 & 405 & 70 & 828 & 1049 & 4281 & 482 & 121 & 61 & 7464 \\
2010 & 259 & 595 & 53 & 712 & 871 & 3770 & 435 & 256 & 49 & 7000 \\
2011 & 280 & 502 & 66 & 396 & 1120 & 3008 & 865 & 141 & 40 & 6418 \\
2012 & 305 & 523 & 27 & 269 & 757 & 1741 & 773 & 124 & 52 & 4571 \\
2013 & 221 & 583 & 23 & 403 & 1139 & 2346 & 932 & 170 & 74 & 5891 \\
2014 & 309 & 500 & 31 & 257 & 1075 & 1887 & 684 & 219 & 50 & 5012 \\
2015 & 264 & 712 & 25 & 209 & 1601 & 2153 & 1030 & 156 & 57 & 6207 \\
2016 & 372 & 1129 & 17 & 258 & 1489 & 2992 & 1376 & 202 & 58 & 7893 \\
2017 & 57 & 1001 & 24 & 265 & 1561 & 2433 & 1243 & 132 & 31 & 6947 \\
Total & 4390 & 9959 & 584 & 7850 & 39890 & 56172 & 19718 & 3079 & 1195 & 143037 \\
\hline
\end{tabular}

Esta análise aponta para a necessidade de se compreender melhor as causas e efeitos deste desmatamento na escala dos estados, considerando que de acordo com as particularidades de cada unidade federativa, os indutores e controladores desse processo trarão influência da matriz econômica interna, do modelo energético predominante na escala local, da estrutura pública dos órgãos de controle, inclusive do modelo de ocupação do território e das relações construídas da população com o espaço.

Compreender as dinâmicas territoriais que induzem esse processo é de fundamental importância se analisarmos tal estudo a partir de uma lógica geossitémica ou corre-se o risco de reproduzirmos o que Armond e Afonso (2011) apontam como os riscos de a matematização ter se tornado tão proeminente no campo destes estudos, explicando a realidade geográfica de forma descritivamente matemática, apresentando assim dificuldades em se relacionar de forma integradora com outros elementos.

Conferindo um zoom na área de análise temos a matriz de transição que avalia o observado e o simulado para o ano final a partir de do cruzamento /dos dados do período de 1997 a 2003 e pode ser observada na Figura 2. 
Figura 2: Observado e Simulado para 2003

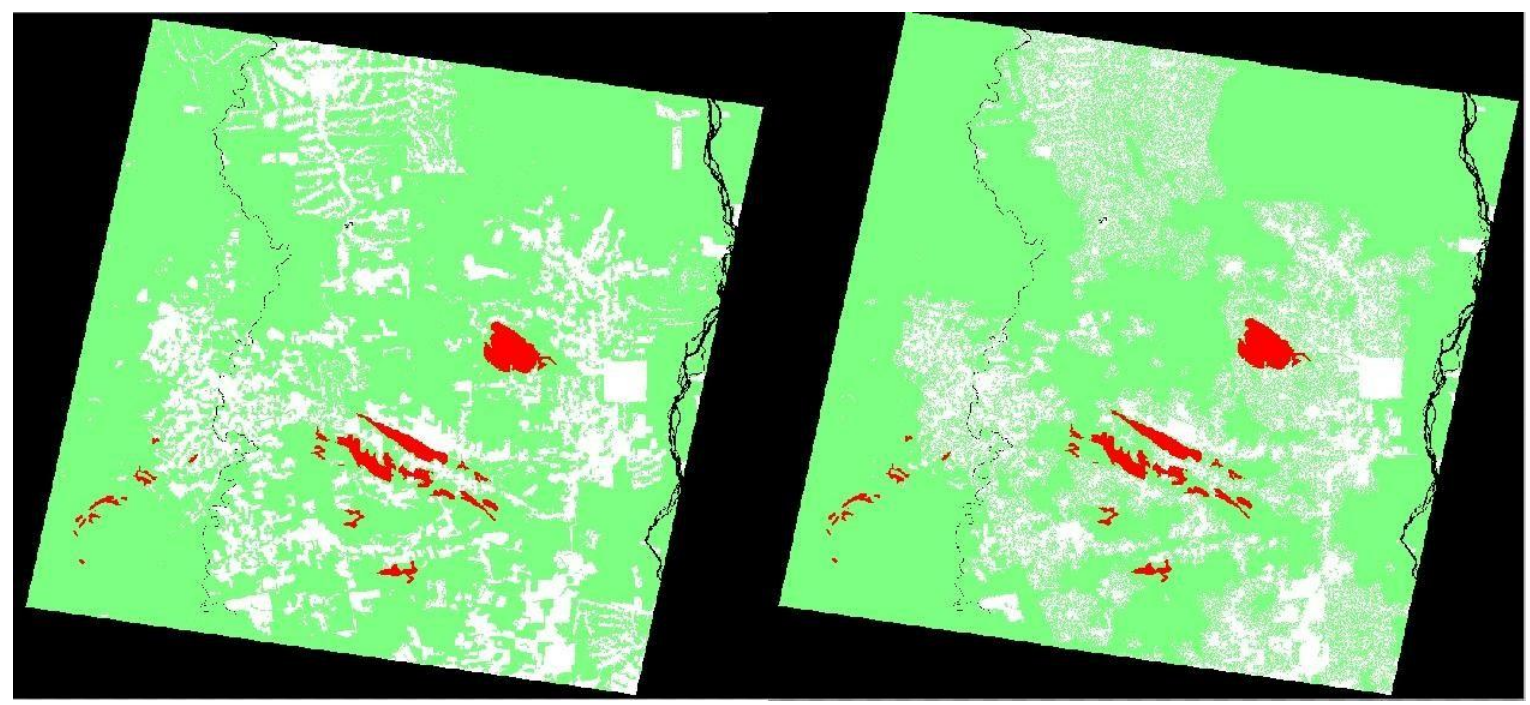

Fonte: Autores

Neste processo foi avaliada através do teste de Crammer a correlação entre as variáveis, sendo que aquelas que apresentaram correlação maior do que 0,5 foram excluídas do modelo. As rodovias pavimentadas e os assentamentos apresentaram Crammer de 0,55, sendo esse valor a maior correlação entre encontrada, desta forma optou-se pela exclusão da variável assentamento para elaboração e calibração do modelo, uma vez que a variável rodovia possuía maior expressividade espacial.

A análise revelou uma taxa de mudança no uso da terra pelo desmatamento de aproximadamente $15 \%$ no período total em análise e aproximadamente $2 \%$ ao ano, enquanto no período analisado com os dados do PRODES houve uma variação média de mudança por meio do desmatamento de aproximadamente 13\%, observam-se então dados semelhantes à taxa de mudança do uso por desmatamento.

Melo (2009) destaca que a mesorregião norte mato-grossense é a maior de todas tendo uma ampla área para cultivo agrícola. E ainda as atividades agroindustriais também crescem na região como mecanismo de beneficiamento e diversidade da economia local. Sendo que a expansão da fronteira agrícola e o crescimento da urbanização justificam o alto índice de na mudança da terra por desmatamento.

Outro ponto importante a observar na imagem que o modelo para 2003 apresenta diferença visual na classe de desmatamento ao observado no mesmo ano, sendo que o estado do Mato Grosso representa o maior produtor de soja no Brasil. 
Neste ponto Schlesinger e Noronha (2006) ao analisar a área de floresta desmatada para os anos de 2003 e 2004 observaram um aumento de 6\% com relação ao período anterior o que demonstra uma forte indicação do potencial do desmatamento.

Os resultados do índice de similaridade furæy podem ser observados na Tabela 1.

\begin{tabular}{|c|c|}
\hline Tamanho das Janelas & Mínima Similaridade \\
\hline $1 \times 1$ & 0,18 \\
\hline $3 \times 3$ & 0,60 \\
\hline $5 \times 5$ & 0,74 \\
\hline $7 \times 7$ & 0,78 \\
\hline $9 \times 9$ & 0,81 \\
\hline $11 \times 11$ & 0,83 \\
\hline
\end{tabular}

Tabela 1: Índice de Similaridade furay

Nota-se que quanto maior a janela maior será a similaridade, assim a repsectiva análise demonstra um alto poder de explicação do modelo como pode ser visto na Figura 3 , já que a taxa mínima de similaridade entre o observado e o simulado para a janela de 11x11 é de 0,83, indicando uma boa calibração das variáveis.

Figura 3: Similaridade entre o simulado (2003) e o observado (2003)

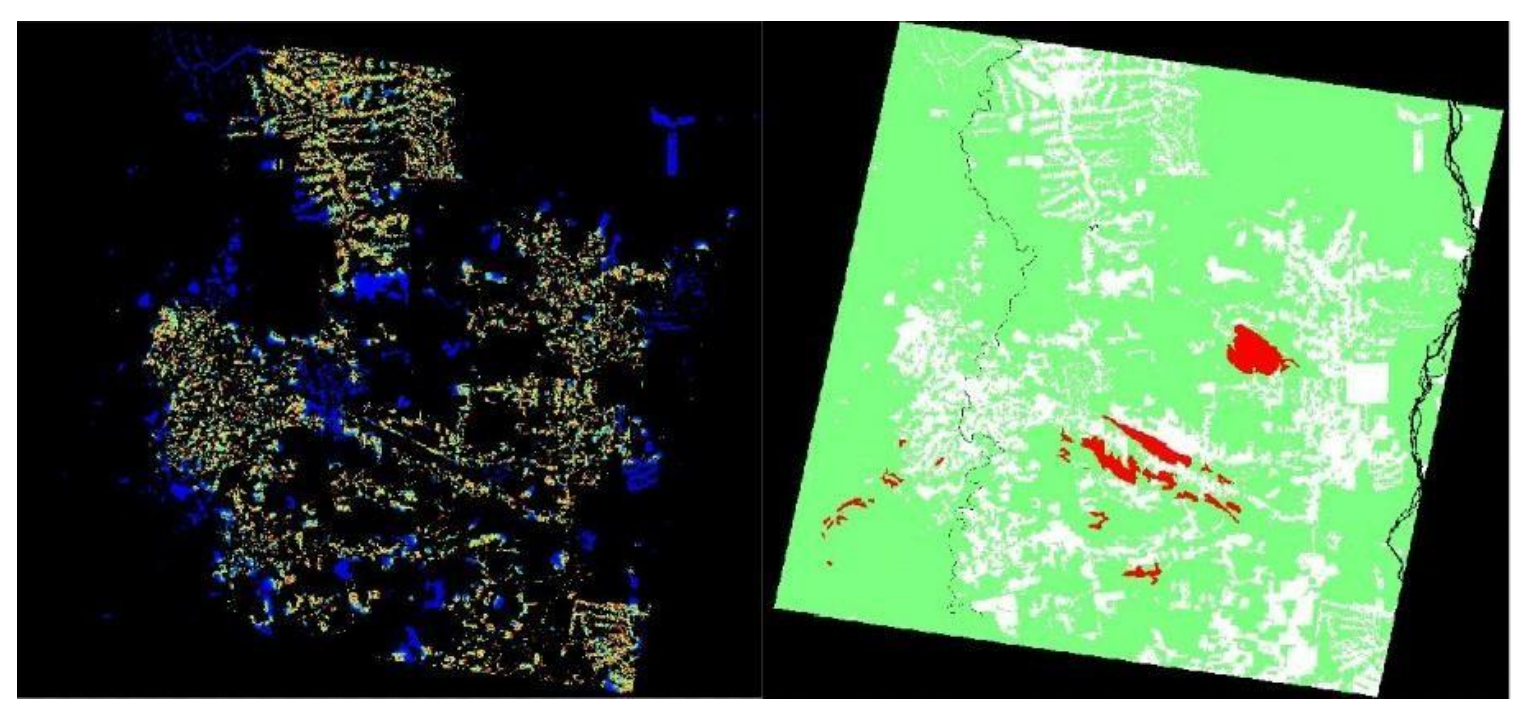

Fonte: Autores 
$\mathrm{Na}$ figura da esquerda as áreas em amarelo e laranja são as áreas que apresentam maior similaridade, enquanto as áreas em azul apresentam menor similaridade. Desta forma, pode-se compreender melhor as possibilidades que o modelo tem de acerto e erro com relação à direção do desmatamento.

A modelagem permite avaliar então a o aumento do desmatamento na área em estudo em uma escala temporal a uma taxa de variação esperada anteriormente calculada e com forte indicação das regiões onde existe a maior possibilidade do modelo obedecer o padrão observado na série histórica de dados, subsidiando assim as tomadas de decisão que poderão mitigar ou eliminar a possibilidade de ocorrência dos impactos advindos dessas alterações.

O produto do modelo preditivo da evolução espaço-temporal do desmatamento entre 2003 e 2033 pode-se observado na Figura 4.

Figura 4: Modelo Evolução espaço-temporal do desmatamento de 2003 a 2033

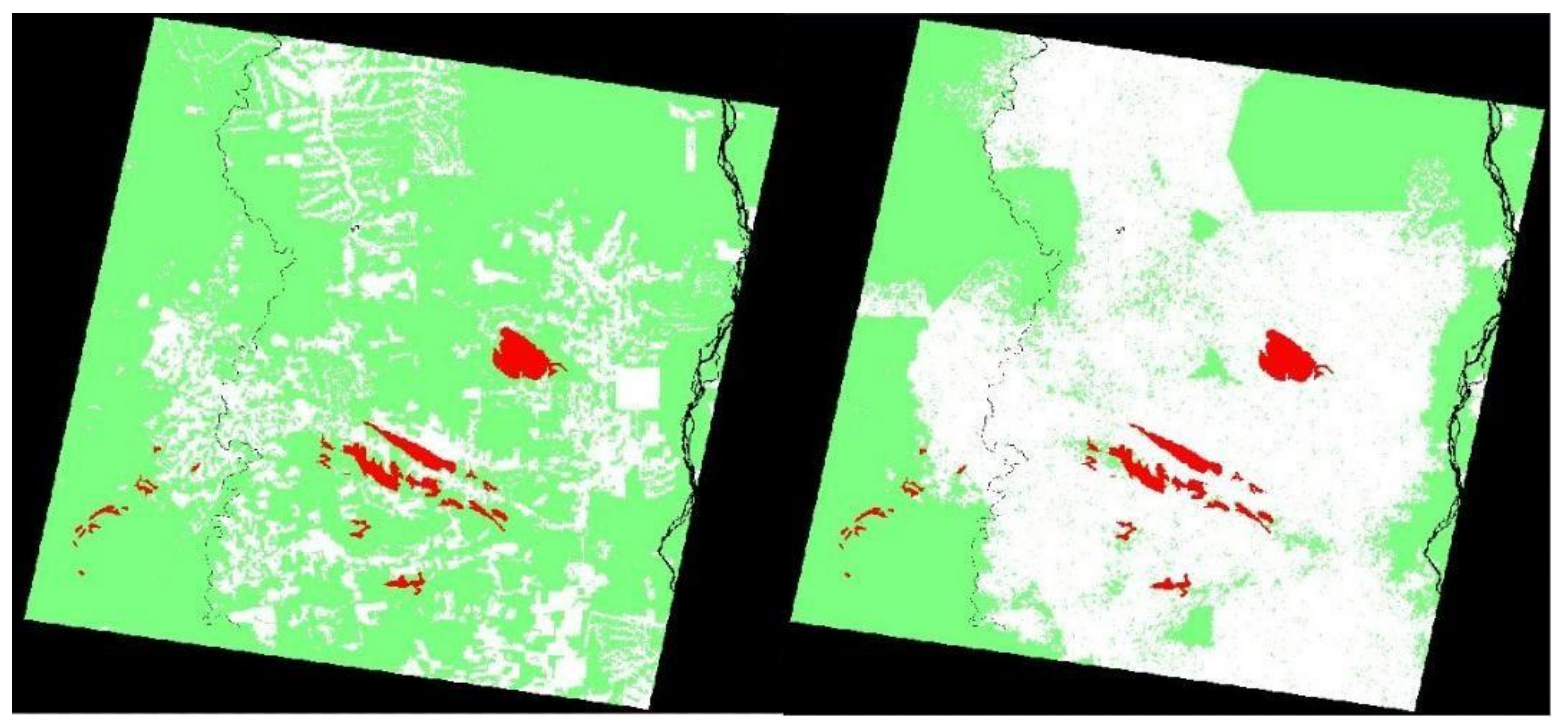

Fonte: Autores

Como pode ser visto acima a área desmatada pode crescer expressiva e significativamente no cenário esperado para 2033, nota-se que um adensamento do desmatamento nas áreas de fragmentos já desmatados e sua expansão pro entorno, além de uma tendência de concentração ao centro da cena principalmente nas áreas próximas as manchas urbanas principais.

Soares-Filhos (2005) ao modelar cenários futuros de desmatamento na região norte do Mato Grosso encontrou dados semelhantes apontando para a continuidade da trajetória do último período por ele analisado podendo acarretar próximo ao ano de 2025 numa redução para cerca de um quinto de extensão original da floresta na área. 
Pereira et al. (2018) apontam que as áreas florestais no Mato Grosso e Pará apresentam pouca valorização e baixo preço tanto da terra nua quanto da superfície dotada de uso florestal, sendo que nas regiões mais produtivas do Mato Grosso, como, Sinop, Sorriso, dentre outras, as áreas florestadas podem chegar a valor próximo de zero em comparação as áreas de florestas derrubadas dentro de uma mesma propriedade. Essa informação indica o que pode ser um importante agende indutor do desmatamento.

\section{4 - CONSIDERAÇÕES FINAIS}

A modelagem de dinâmica da paisagem por simulação de cenários se apresenta como uma importante ferramenta de análise da mudança em cobertura do solo, expressando grande potencial de subsidio as tomadas de decisão e a formulação de políticas e mecanismos de controle e mitigação de impactos.

Tal análise sem a capacidade de contextualização e sem as devidas considerações quanto ao seu recorte geográfico corre o risco de não representar efetivamente os fenômenos relacionados à sua causalidade.

A evolução do pensamento geográfico a partir da geografia quantitativa possibilitou o desenvolvimento de um conjunto de ferramentas que representam uma infinidade de oportunidades de análise ambiental.

É fundamental o conhecimento da historicidade dos métodos científicos adotados e seu aporte teórico filosófico para garantir a utilização de maneira consciente, considerando inclusive suas limitações. 


\section{REFERÊNCIAS BIBLIOGRÁFICAS:}

ARMOND, B. N.; AFONSO, A. E. A GEOGRAFIA TEORÉtTICO-QUANTITATIVA E A TEORIA DOS GEOSSISTEMAS: POR UMA GEOGRAFIA FÍSICA INTEGRADORA. XIV Simpósio. Bras. Geografia Física Aplicada SBGFA 1, 1-12

CHRISTOFOLETTTI, Antonio. As perspectivas dos estudos geográficos. In: (org.) Perspectivas da Geografia. $2^{a}$ edição. São Paulo: DIFEL, 1985.

Environmental Systems Research Institute (ESRI). Arcgis for Desktop. Versão. 10.3. Redlands: ESRI, 2013.

FRANÇA, Marcos Tertuliano. Análise do crescimento do volume da produção agrícola na região norte mato-grossense e sua relação com os problemas de solo no período de 1980 a 200 . Cuiabá MT - 2009. xvi, $121 \mathrm{f}$.

MELO, J. S. Qual é o determinante da expansão da fronteira agrícola mato-grossense, no período de 2001/2007: Produção Agrícola ou Pecuária?. Cuiabá - MT, 2009.

PEREIRA, M. P. R.; Tisler, T. R.; Nobrega, R. A. A. Monetização de Superfície - Reflexões sobre o preço da terra na área do corredor modelado para Ferrogrão no Mato Grosso e Pará. (no prelo).

SCHLESINGER, S.; NORONHA, S. O Brasil está nu! O avanço da monocultura da soja, o grão que cresceu demais. Rio de Janeiro: FASE, 2006.

SOARES-FILHO, B. S.; CERQUEIRA, G. C.; PENNACHIN, C. L. DINAMICA - a stochastic cellular automata model designed to simulate the landscape dynamics in an Amazonian colonization frontier. Ecological Modelling, v. 154, p. 217-235. 2002.

SOARES-FILHO, B. S. Análise das mudanças de cobertura do solo no Norte do Mato Grosso, Brasil. Anais XII Simpósio Brasileiro de Sensoriamento Remoto, Goiânia, Brasil, 16-21 abril 2005, INPE, p. 3357-3364.

SOUZA, E. B. C. Estado: produção da região do Lago de Itaipu - turismo e crise energética. Tese (Doutorado em Geografia) - Presidente Prudente: UNESP. 2002. 